\title{
Automated Gait Analysis to Assess Functional Recovery in Rodents with Peripheral Nerve or Spinal Cord Contusion Injury
}

\author{
Johannes Heinzel ${ }^{1,2,3}$, Nicole Swiadek ${ }^{2,3}$, Mohamed Ashmwe ${ }^{2,3}$, Alexander Rührnöß1 ${ }^{2,3}$, Viola Oberhauser ${ }^{2,3}$, Jonas \\ Kolbenschlag ${ }^{1}$, David Hercher ${ }^{2,3}$ \\ ${ }^{1}$ Department of Hand-, Plastic, Reconstructive and Burn Surgery, BG Trauma Center Tubingen, Eberhard Karls University ${ }^{2}$ Ludwig Boltzmann Institute for \\ Experimental and Clinical Traumatology ${ }^{3}$ Austrian Cluster for Tissue Regeneration
}

\section{Corresponding Author}

David Hercher

david.hercher@trauma.lbg.ac.at

\section{Citation}

Heinzel, J., Swiadek, N., Ashmwe, M., RührnößI, A., Oberhauser, V., Kolbenschlag, J., Hercher, D. Automated Gait Analysis to Assess Functional Recovery in Rodents with Peripheral Nerve or Spinal Cord Contusion Injury. J. Vis. Exp. (164), e61852, doi:10.3791/61852 (2020).

\section{Date Published}

October 6, 2020

DOI

$10.3791 / 61852$

URL

jove.com/video/61852

\section{Abstract}

Peripheral and central nerve injuries are mostly studied in rodents, especially rats, given the fact that these animal models are both cost-effective and a lot of comparative data has been published in the literature. This includes a multitude of assessment methods to study functional recovery following nerve injury and repair. Besides evaluation of nerve regeneration by means of histology, electrophysiology, and other in vivo and in vitro assessment techniques, functional recovery is the most important criterion to determine the degree of neural regeneration. Automated gait analysis allows recording of a vast quantity of gait-related parameters such as Paw Print Area and Paw Swing Speed as well as measures of inter-limb coordination. Additionally, the method provides digital data of the rats' paws after neuronal damage and during nerve regeneration, adding to our understanding of how peripheral and central nervous injuries affect their locomotor behavior. Besides the predominantly used sciatic nerve injury model, other models of peripheral nerve injury such as the femoral nerve can be studied by means of this method. In addition to injuries of the peripheral nervous systems, lesions of the central nervous system, e.g., spinal cord contusion can be evaluated. Valid and reproducible data assessment is strongly dependent on meticulous adjustment of the hard- and software settings prior to data acquisition. Additionally, proper training of the experimental animals is of crucial importance. This work aims to illustrate the use of computerized automated gait analysis to assess functional recovery in different animal models of peripheral nerve injury as well as spinal cord contusion injury. It also emphasizes the method's limitations, e.g., evaluation of nerve regeneration in rats with sciatic nerve neurotmesis due to limited 
functional recovery. Therefore, this protocol is thought to help researchers interested in peripheral and central nervous injuries to assess functional recovery in rodent models.

\section{Introduction}

Injuries of the peripheral and central nervous system are often studied in rodents, resulting in a great amount of comparative data regarding the course of nerve injury, repair, or neuroprotection to counteract further secondary injuries and regeneration ${ }^{1,2,3}$. The outcome of experimental treatment strategies in rodent models can be assessed by a variety of techniques such as histology, immunohistochemistry, electrophysiology, and imaging techniques such as X-ray microtomography $(\mu \mathrm{CT})$ scans, but the most important criterion to determine the success of a treatment is-like in human patients-the degree of functional recovery ${ }^{4,5}$. The first studies investigating locomotor performance in rodents date back to the $1940 s^{6,7,8}$. Rats and mice were subject to a great amount of studies investigating their locomotor behavior in the following decades $9,10,11$. Nowadays, a broad range of assessment techniques for rodent models of peripheral and central nerve injuries exist, ranging from walking track analysis with ink and paper ${ }^{12,13,14}$ over ankle and gait kinematics ${ }^{15,16,17}$ to machine-learning enhanced methods, which allow for the complex estimation of gait, limb, and joint trajectories $^{18,19}$.

Computerized Automated Gait Analysis (AGA) is used to evaluate locomotor function following peripheral and central nervous injuries and potential experimental treatment of such injuries. The device mainly consists of a glass walkway and a light source that illuminates the rodent's paw prints in correlation with the pressure exceeded by them. This data is then computerized to calculate a broad array of static and dynamic parameters. According to Deumens, these parameters can be further subdivided into the categories of general parameters, pain-related parameters as well as coordination-related parameters of gait ${ }^{20}$ (Table 1). The feasibility of AGA to detect changes in gait behavior has been proven in various animal models of peripheral nerve injury $(\mathrm{PNI})^{21}$, such as the sciatic nerve ${ }^{20}$, femoral nerve ${ }^{22}$, and median nerve 23,24 . It is also routinely used to assess locomotor function in rats with central nervous injuries, e.g., stroke $^{25}$ or spinal cord contusion ${ }^{26}$. The method's advances lie in the great amount of comparable data and its possibility to record a plethora of parameters related to gait ${ }^{27}$. This paper aims to provide researchers interested in animal models of $\mathrm{PNI}$ and spinal cord injury (SCI) with a detailed and hands-on guideline to assess locomotor function in such models.

\begin{tabular}{|c|c|c|}
\hline Category & Parameter & Description \\
\hline General parameters of gait & Print Area (distance unit) & Area of the paw print \\
\cline { 2 - 3 } & Print Length (distance unit) & Length of the paw print \\
\cline { 2 - 3 } & $\begin{array}{c}\text { Base of Support } \\
(\text { BoS }) \text { (distance unit) }\end{array}$ & Distance between the two hind-or front paws \\
\hline
\end{tabular}




\begin{tabular}{|c|c|c|}
\hline & Stride Length (distance unit) & Distance between two consecutive placements of a paw \\
\hline \multirow{3}{*}{$\begin{array}{l}\text { Pain-related } \\
\text { parameters of gait }\end{array}$} & Swing Time (s) & Duration of the swing phase \\
\hline & Stand Time (s) & Duration of the stance phase \\
\hline & $\begin{array}{c}\text { Mean Paw Print } \\
\text { Intensity (arbitrary unit) }\end{array}$ & Mean ilntensity of the paw print during the stance phase \\
\hline \multirow[t]{3}{*}{$\begin{array}{l}\text { Coordination-related } \\
\text { parameters of gait }\end{array}$} & $\begin{array}{l}\text { Normal Step Sequence } \\
\text { Patterns (NSSP) }\end{array}$ & Specific sequences of paw placements during a step cycle \\
\hline & Phase Dispersions (\%) & $\begin{array}{l}\text { Temporal differences between the } \\
\text { step cycles of two specific paws }\end{array}$ \\
\hline & Regularity Index (RI) (\%) & $\begin{array}{l}\text { Quantification of interlimb coordination by dividing } \\
\text { the amount of flawless NSSP times } 4 \text { by the overall } \\
\text { number of paw placement during one step cycle }\end{array}$ \\
\hline
\end{tabular}

Table 1: Parameters of gait assessable with the automated gait analysis. The categories in which the parameters are classified are chosen according to Deumens et al. ${ }^{20}$.

\section{Protocol}

The experimental protocol for all experiments was approved in advance by the Animal Protocol Review Board of the City Government of Vienna. All procedures were carried out in full accordance to the Helsinki Declaration on Animal Rights and the Guide for the Care and Use of Laboratory Animals of the National Institutes of Health.

\section{Animal housing}

1. House male rats (Lewis or Sprague Dawley) weighing 250-300 g under a $12 \mathrm{~h}$ light/dark cycle with ad libitum access to food and water.

2. Control both room temperature (maintained at $20-22{ }^{\circ} \mathrm{C}$ ) and humidity (45\%-65\%) for adequate animal housing.
For this work, male Lewis (PNIs) and Sprague Dawley $(\mathrm{SCl})$ rats were used.

3. Provide a new, cleaned cage twice per week. House rats in groups of two or three and closely monitor their social behavior and interaction. Allow the rats an acclimation period of at least 1 week before any surgical procedure or functional testing.

NOTE: Rats require at least 5 days of daily training on the gait analysis apparatus prior to surgery, so calculate at least 2 weeks between arrival of the rats at the facility and the scheduled data for experimental surgery ${ }^{28}$.

\section{Induction of nerve injury}

NOTE: Wear personal protective equipment such as a surgical gown, gloves, and a mask. In case a sterile surgical gown is not available, a clean, laundered lab coat is also sufficient. Unless it becomes contaminated, the gown or 
coat does not need to be changed between animals but between surgery sessions. The use of sterile surgical gloves is recommended. In case such gloves are not available, examination gloves can also be used but should be washed prior to surgery using surgical disinfectant. Gloves should be changed between animals.

1. On the day of surgery, try to minimize any amount of stress to the animals as this might interfere with the anesthesia, e.g., animals will require higher doses of anesthetics.

2. On the day of surgery, inject $0.05 \mathrm{mg}$ Buprenorphine/kg bodyweight in suspension with $100-200 \mu \mathrm{L} 0.9 \% \mathrm{NaCl}$ subcutaneously using a $25 \mathrm{G}$ canula $1 \mathrm{~h}$ prior to surgery to provide pre- and interoperative analgesia. If not indicated otherwise, the rat's flank is the preferred site for injection.

3. Anesthetize the rats by placing them in an anesthesia induction chamber connected to a sevoflurane vaporizer and an active chalk container to absorb the $\mathrm{CO}_{2}$. Anaesthetize the rat by flooding the anesthesia box with $4 \%-5 \%$ sevoflurane-oxygen mixture for about 5 min using an oxygen flow of $1.5 \mathrm{~L} / \mathrm{min}$ (initiation phase). Connect a pulse oximetry clip to one of the paws to monitor the rodent's blood oxygen saturation. Maintain the state of general anesthesia with $2.5 \%-4.5 \%$ sevoflurane-oxygen mixture.

NOTE: For experimental surgery, general anesthesia is obligatory. Confirm general anesthesia by checking the lack of response to pinch stimulus of the tail or paws.

4. When the rat has entered general anesthesia, shave the respective areal that is going to be operated on and disinfect the area by swiping it alternatingly with alcohol and skin disinfectant. The final swiping should be performed with skin disinfectant.
5. Place it on an adjustable heating pad in the required position (supine for the femoral nerve model, prone for the sciatic and $\mathrm{SCl}$ model). Insert a flexible thermometer probe in the rat's rectum to monitor the animal's temperature and maintaining it at around $37^{\circ} \mathrm{C}$ during surgery. During anesthesia, protect the rat's eyes from desiccation using eye ointment

\section{Surgical induction of nerve injury}

1. When performing surgical procedures, adhere strictly to the seven principles of Halsted ${ }^{29}$ as follows:

1. Always handle the tissues gently when working with them. Avoid tearing or crushing the tissues.

NOTE: Self-made ${ }^{30}$ or commercially available retractor systems are helpful to keep muscles and vessels out of the operating field.

2. Carefully maintain the hemostasis using an electric hemostat to cauterize vessels or ligatures to guarantee careful hemostasis.

3. Always maintain blood supply to the tissues by dissecting them carefully and handling them delicately.

4. Maintain strict asepsis by wearing a mask, a gown, and sterile gloves.

5. Avoid tension on the tissues by applying sutures which are neither too tight nor too loose.

6. Meticulously appose the tissues by bringing their respective edges together without any overlapping. NOTE: This is particularly important in the case of epineurial or perineurial neurorrhaphy. Perform all microsurgical procedures under $6 x-16 x$ magnification using an operating microscope. Ideally, 
the microscope provides two pairs of oculars to allow for observation of the operation by an assistant.

7. Avoid creation of dead spaces by carefully suturing the different tissue layers.

2. Induction of sciatic nerve neurotmesis of the right hindlimb

1. Expose the right sciatic nerve at the midthigh level by performing a $5 \mathrm{~cm}$ long incision on the dorsal side of the hind limb with a \#10 scalpel blade connected to a \#3 scalpel handle and by dissecting the overlying musculature and soft tissue. Place a retractor inside the wound to keep the dissected musculature and skin out of the situs. Gently expose the nerve by removing the surrounding tissue using curved microsurgical scissors.

2. Remove an $8 \mathrm{~mm}$ long nerve segment of the sciatic nerve $1-2 \mathrm{~mm}$ proximal to its trifurcation with straight microsurgical scissors.

3. Rotate the nerve segment $180^{\circ}$, place it between the proximal and distal stump of the transected sciatic nerve and perform epineurial neurorrhaphy at each site with two interrupted $10-0$ sutures and a microsurgical needle holder.

3. Induction of femoral nerve neurotmesis on the right hindlimb

1. Perform a longitudinal $3-4 \mathrm{~cm}$ groin incision with a \#10 scalpel blade connected to a \#3 scalpel handle in order to expose the right femoral neurovascular bundle. Use surgical scissors for blunt dissection until the bifurcation of the femoral nerve is exposed. Place a retractor inside the wound to keep the dissected musculature and skin out of the situs.
2. Transect the exposed motor and sensory branches distal to the bifurcation and excise a $6 \mathrm{~mm}$ long nerve segment of each branch, respectively, using straight microsurgical scissors.

3. Rotate both nerve segments $180^{\circ}$, place them between the proximal and distal stump of the transected femoral nerve branches and perform epineurial neurorrhaphy at each site with two interrupted $11-0$ sutures and a microsurgical needle holder.

NOTE: Perform a homotopic autologous nerve graft by grafting the motor graft to the original motor branch and the sensory graft to the original sensory branch. Alternatively, perform a heterotopic autologous nerve graft by grafting the motor graft to the original sensory branch and vice versa.

\section{Induction of thoracic spinal cord contusion injury}

1. Perform a skin incision across the thoracic spinal column with a \#10 scalpel blade connected to a \#3 scalpel handle followed by two muscle incisions parallel to the spinous processes to facilitate muscle retraction. Place a retractor inside the wound to keep the dissected musculature and skin out of the situs.

2. Identify the 11th thoracic vertebra (Th) and expose the lamina of the vertebral arch by removing overlying tissue as well as the spinous process using a rongeur.

3. Perform a laminectomy using a micro drill and an appropriate burr to drill a small hole into the lamina, slightly larger than the tip of the impactor. To prevent damage to the spinal cord, only thin out the lamina while opening and enlarging the hole using a rongeur. If the periosteum is still intact, carefully remove it using 
a sharp pointed probe without damaging the dura mater.

4. After ensuring a large enough hole is made without destabilizing the lamina, hold the animal's spinal column in place by clamping it rostrally and caudally to Th11 with the impactor's stabilizing forceps. Using the hand wheels at the front and the side, position the steel rod $3-5 \mathrm{~mm}$ above the laminectomy hole. Finally, subject all animals to an impact with a defined force of 150 kilodyne to induce a mild to moderate degree of spinal cord contusion injury ${ }^{4}$.

5. Perform wound closure in anatomical layers using polyglactin $4-0$ or $5-0$ interrupted sutures and a surgical needle holder. Clean the wound thoroughly by gently wiping it with a gauze pad soaked in sterile $0.9 \% \mathrm{NaCl}$.

6. After surgery, return the animals to their home cage and shelter them from light and sound exposure. Monitor the animals' behavior closely until postoperative day (DPO) 7 and assure sufficient food and water intake. If needed, provide additional fluid by subcutaneous injections (e.g., $10 \mathrm{ml} \mathrm{NaCl} \mathrm{0.9 \% ).}$

7. Provide post-operative analgesia for a minimum of 2 days by means of, e.g., opioids $(0.05 \mathrm{mg} /$ kg bodyweight Buprenorphine subcutaneously (s.c.)) and/or antipyretics $(4 \mathrm{mg} / \mathrm{kg}$ bodyweight Carprofen s.c.). If required, and in the case of the $\mathrm{SCl}$ model, also provide postoperative antibiotic therapy $(7.5 \mathrm{mg} /$ kg bodyweight Enrofloxacin per os (p.o.)).

8. In case of spinal cord injury, manually empty the rat's bladder until spontaneous urination returns.

\section{Recovery from surgical interventions prior to gait analysis}

NOTE: Rats with sciatic nerve injury show the tendency to gnaw on their hind paws due to onset of painful neuropathy following nerve injury. This form of automutilation can result in autoamputation of toes or parts of the respective hind paw. Prefer Lewis rats over other rat strains in case when using the sciatic nerve injury model, as this rat strain shows a smaller tendency for automutilation ${ }^{31}$. Rats with sciatic nerve injury also show the tendency to develop contractures of the operated limb, which might result in their exclusion from the study due to interference with data acquisition. Such adverse events occur far less common in rats with femoral injury.

1. Inspect operated animals daily following surgery with particular attention on the status of their limbs and paws, respectively.

NOTE: In rats with $\mathrm{SCl}$, at the height of Th11, penile or rectal prolapse might can occur due to the impairment of the animals' natural capability to urinate and defecate. These events are commonly defined as human endpoints of the study and imply immediate exclusion of the affected animal from the study.

2. Continue postoperative analgesia until rats cease to display any pain-related symptoms.

3. In case of persistent pain, administer gabapentin (30-120 $\mathrm{mg} / \mathrm{kg}$ bodyweight) p.o. to treat neuropathic pain.

\section{Preparation prior to performing automated gait analysis}

NOTE: The gait analysis system's methodology is based on recording the animals from below while crossing a glass plate, which is illuminated by a green LED light. When the animals' paws contact the floor, the area of the paw print is illuminated 
and recorded by a high-speed video camera. This data is then sent via an Ethernet cable to a computer running the gait analysis software. While individual footprints can be classified manually by the experimenter, the latest software version also features automatic footprint classification.

1. Perform all testing procedures in the dark and in the absence of disturbing noises. Since rats are able to perceive supersonic frequencies, also verify that no sources are emitting such sounds.

NOTE: Perform gait analysis either weekly or every other week, but do not test rats too frequently as especially Lewis rats tend to lose interest in participating in the procedure over time when they are exposed to a certain exercise too frequently. It is, however, required to train rats daily for 5 days prior to surgery to acclimate them to the testing environment and procedure.

2. During training sessions and on the test day, prepare the behavioral test room by turning off all light sources, which could otherwise interfere with the automated gait analysis device's camera. Place the computer screen necessary for data acquisition away from the camera to prevent its light from interfering with the camera.

3. Make sure the device is installed in a stable position and in a way that prevents any form of vibration, as this will seriously interfere with the data acquisition procedure.

4. Bring the rats to the behavioral test room and acclimate them in their own home cage for at least 30 min before the test.

NOTE: Whenever handling the animals, wear personal protective equipment such as a surgical gown or lab coat, gloves, and a mask.

\section{Performing automated gait analysis}

1. Training sessions

NOTE: During training, animals will undergo a learning curve, so it is recommended to adjust the training schedule gradually. Use food rewards (e.g., 1-2 pieces of breakfast cereal) to reward the animals after successful completion of each training session.

1. On the first day of training, gently lift the animal by holding it underneath its trunk and gently carry it to the walkway entrance.

2. Place the animal in the entrance area and let it explore the opening of the corridor without any interference from the person carrying out the testing procedure.

NOTE: Do not scream, whistle, blow at or poke the animal in an attempt to motivate it to cross the walkway. All such behavior will severely stress the animal and further complicate the data acquisition procedure.

3. Wait until the animal voluntarily crosses the walkway to reach its home cage. Sometimes, especially in untrained animals, this can take up to several minutes. On the first training day, the animal is neither expected nor required to make uninterrupted runs with uniform walking speed. Instead it should acclimate itself with the testing apparatus and procedure.

4. On the second day of training, accustom animals to enter the walkway without hesitation and, also to return to their home cage without hesitation. Some animals will probably already have learned to cross the walkway without interruptions, but this is still not required at the end of the second day. 
5. On the third day of training, ensure animals learn to cross the walkway without hesitation, sniffing, or otherwise explorative movements. Ensure they walk with uniform speed.

6. On the fourth and fifth day of training, repeat the previous exercise to consolidate the testing procedure.

NOTE: In case an animal does not acquire the necessary skill to cross the walkway properly by the end of the 5-day training period, add 2 additional days of training (e.g., the weekend). Also, consider performing up to 3 training sessions a day, separated by at least $2 \mathrm{~h}$ of resting between individual session. In $95 \%$ of the cases, the animal will have acquired the required training experience by the end of this extended training period. In the rare case an animal has still not acquired this skill after 7 days of training, it is recommended to postpone the planned experimental surgery session for at least 1 week and to repeat the aforementioned training regime.

\section{Data acquisition}

NOTE: The gait analysis system visualizes each paw print while the animal is walking and automatically analyzes various gait parameters such as Paw Print Area, Paw Print Intensity, Paw Swing Time, and Paw Swing Speed (Table 1). As the gait analysis system records all data based on the intensity generated by the animals' paw prints, make sure the camera settings are adjusted according to the rats' weight and size. Additionally, make sure the walkway is dry and clean prior to data recording to prevent any influence on data acquisition.
1. Before acquisition of any data, clean the walkway using commercial glass cleaner and a squeegee. Spray the glass plate several times and then wipe it with the squeegee to remove any particles from its surface. Also, clean the underneath. Make sure to remove any fluid from the ends of the walkway since the animals could otherwise step on it, which would affect the recorded data.

2. Repeat the cleaning procedure whenever necessary, e.g., contamination of the walkway and before recording of data of a rat from a different cage. This is thought to prevent the animal from being distracted by their conspecifics' scent.

3. Prior to the very first data acquisition, adjust the camera settings fitting the animals' weight. Confirm this by putting the lightest and heaviest animal on the walkway and choose a camera setting that enables good data quality in both cases. Adjust the Camera Gain, Red Ceiling Light, Green Walkway Light, and Green Intensity Threshold (GIT) to ensure optimal paw print detection.

NOTE: Do not change the chosen settings after data acquisition has begun as this will hinder comparability of the acquired data. As an exception, the GIT can be changed during data classification, but this must be done for all trials uniformly.

4. Define and calibrate the walkway using the provided calibration sheet.

5. Choose a registered camera listed in the Setup tab.

6. Click on the Open Acquisition button which can be found in the Acquire tab.

7. Take a snapshot of the empty, cleaned walkway, which will be used as a reference throughout the following data acquisition procedure. 
8. Note the status changing from Waiting for Snapshot to Ready for Acquisition.

9. Click on the Start Acquisition button and notice the status change from Ready for Acquisition to Waiting for

\section{Run to Start.}

10. Place a rat on the walkway and follow the animal's movement on the computer screen. Note the status change from Waiting for Run to Start to Recording Run. NOTE: The software will automatically classify runs it deems compliant according to preset run characteristics with a green symbol, while non-compliant runs will be marked with a red symbol. The software automatically stops data acquisition when three compliant runs were recorded, but data acquisition can be continued by clicking on the Start Acquisition button again.

\section{Data classification}

NOTE: Refer to Table 1 for a list of AGA outcome parameters. At least three compliant runs are needed in which the animal must cross the walkway steadily without hesitation ${ }^{27}$.
Additionally, running speeds should match within the same categories as defined in the literature ${ }^{30}$.

1. Click on the Classify button in the Experimental Explorer tab of the respective trials that are to be classified.

2. Play the acquired run with normal speed to get an impression whether the data conforms to the requirements listed before.

3. In the left-upper corner, click on the Auto Classify button for automatic classification of paw prints by the software. NOTE: Although the software has a high rate of correct paw classification, it sometimes fails to assign a paw to the prints or assigns the wrong paw. Therefore, always double check the auto-classified paw prints afterwards.

4. For correct calculation of Normal Step Sequence Patterns (NSSPs), make sure that the classifying algorithm is not confused by non-visible paw prints, leading to flawed NSSP (Figure 1A). Therefore, only include the paw prints that are detectable while the contralateral paw is also visible for NSSP calculations, e.g., the left front paw (LF) and the right hind paw $(\mathrm{RH})$ (Figure 1B). 


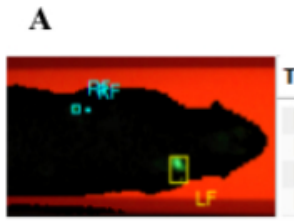

Timing View

Right Front

Right Hind

Left Front

Left Hind

B

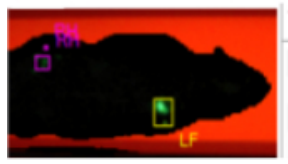

Timing View

Right Front

Right Hind

Left Front

Left Hind

Figure 1: Exemplary AGA data, demonstrating the need for manual double checking of correct data classification. In case a detected placement of a front paw is succeeded by the detected placement of another front paw (A) the AGA software might confuse this with an uncoordinated walking pattern since no hind paws have been detected. Therefore, it is recommended to always double check and select an initial paw print, which is detected when the contralateral paw is also visible (B). Please click here to view a larger version of this figure.

\section{Calculating statistics}

NOTE: To adjust run data for weight gain related changes over time, it is strongly recommended to calculate a ratio of the experimental paw with a non-experimental (e.g., control) paw. Additionally, calculate the ratio to preoperative values of this paw-to-paw comparison for consideration of individual differences in paw use.

1. Click on the View Run Statistics button to get a comprehensive overview on the run statistics.
2. Select File and Export to either export run statistics or trial statistics into a spreadsheet software.

\section{Representative Results}

12 rats underwent experimental peripheral nerve surgery. Sciatic nerve resection (Figure 2A) was performed in 7 rats, while femoral nerve neurotmesis (Figure 2B) was induced in 5 rats. In all animals, the nerve defect was reconstructed by means of an autologous nerve graft. Spinal cord contusion injury (Figure 2C) at level Th11 was induced in 6 rats, resulting in a total of 18 rats. 


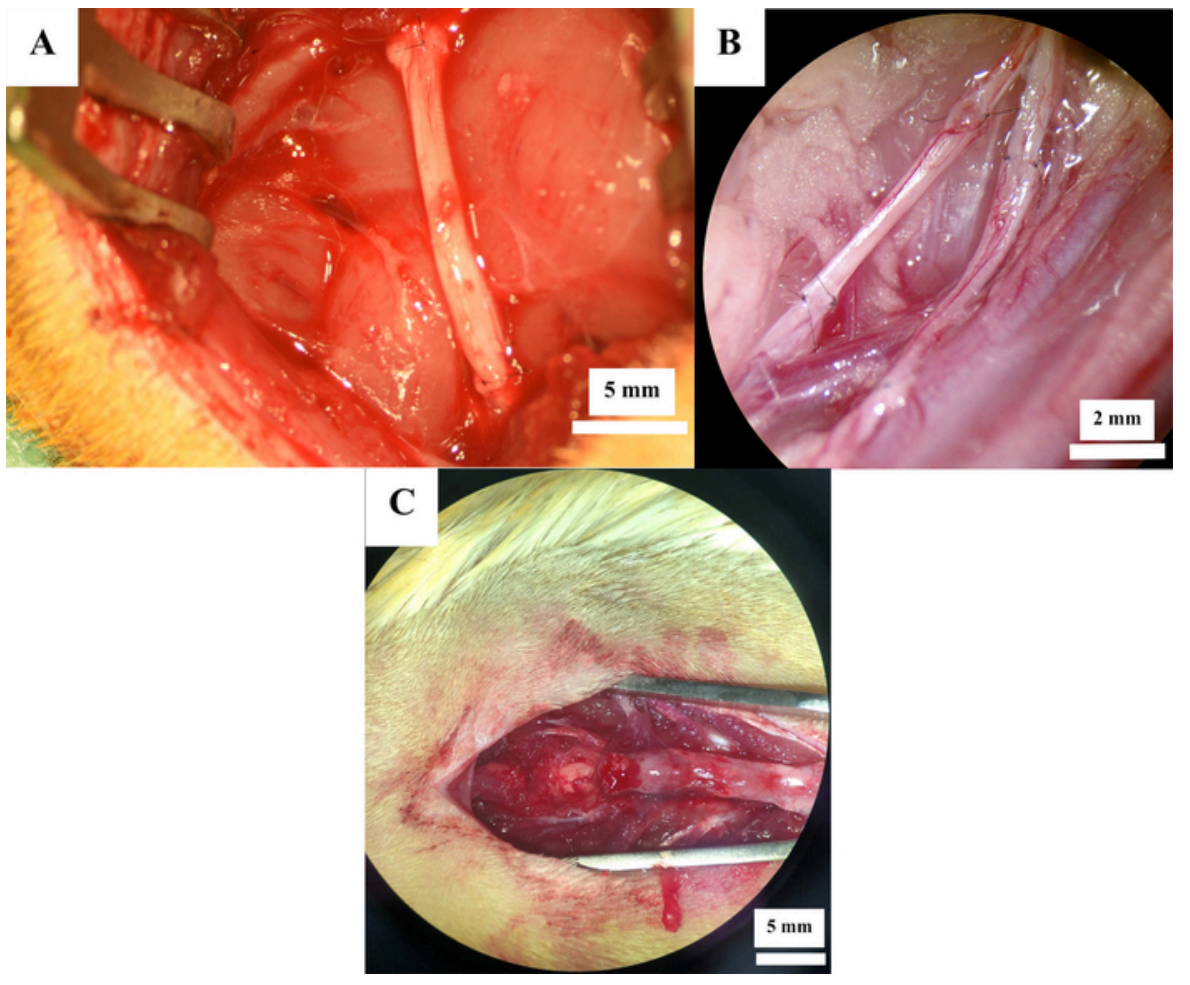

Figure 2: Operative sites after nerve reconstruction. Nerve reconstruction with autografts in the sciatic nerve (A) and femoral nerve (B) as well as after spinal cord contusion injury (C). Please click here to view a larger version of this figure.

All animals recovered well from surgery and no cases of selfmutilation occurred. One animal of the sciatic nerve injury group developed strong contractures of the right hind paw during the course of the postoperative observation period and had to be excluded from further data analysis.

\section{Sciatic nerve neurotmesis}

Since the sciatic nerve provides muscular and sensory innervation to the majority of the hindlimb, its resection results in a severe impairment of locomotor function. Following injury, rats use the heel of the paw for weight support only (Figure 3B-E) and the limb is moved in a sweeping circumductory movement. Therefore, locomotor changes assessed via AGA become apparent by means of a significantly reduced Print Area (Figure 4A) and significantly increased Swing Time (Figure 4B). Both parameters were still significantly altered in comparison to Pre-OP measurements as at the end of the observation period. Noteworthy, one animal developed strong contractures of the right hind paw starting at postoperative week (WPO) 10. This resulted in an increase of the Print Area of the right hind paw to more than $150 \%$ in comparison to the left paw at WPO12 (Figure 5). As this was an extremum in comparison to all other animals assessed in this study, we excluded this animal from data analysis in regard to Print Area. 

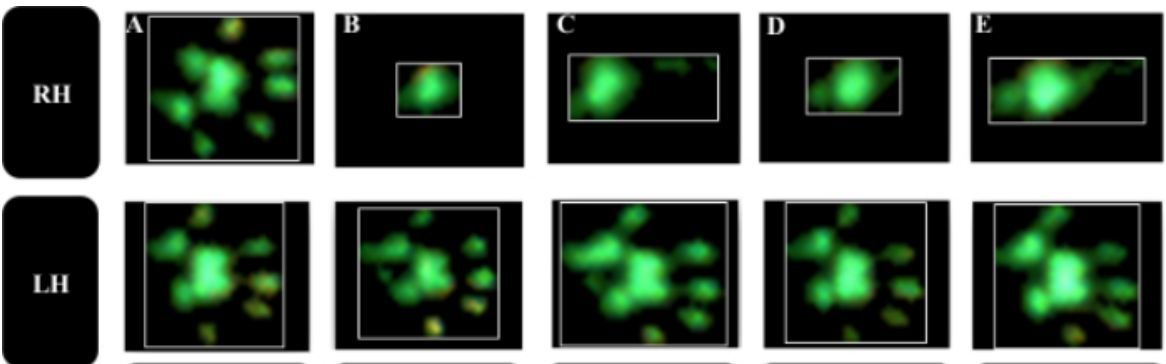

Pre-OP

WPO2

WPO4

WPO8

WPO12

Figure 3: Representative paw prints prior to and following critical size resection of the right sciatic nerve and autograft repair. Note the strong decrease in Print Area following nerve injury (B) as compared to preoperatively (A). Despite a slight increment in Print Area during the course of the observation period (C-E) the paw prints of the right hind limb remained notably changed from baseline recordings. Please click here to view a larger version of this figure.
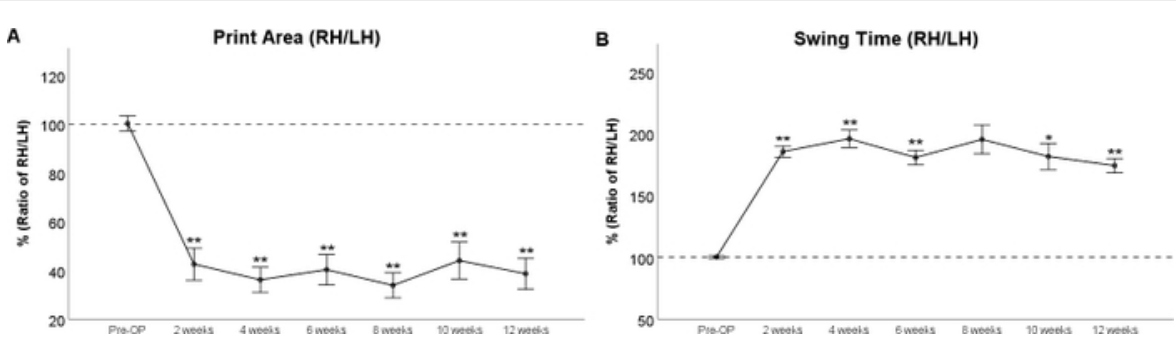

Figure 4: The course of functional recovery after critical size resection and autograft repair of the sciatic nerve. Print Area Ratio (A) and Swing Time Ratio (B) were statistically changed significantly from Pre-OP values immediately after sciatic nerve resection. While Print Area remained significantly decreased compared to baseline until WPO10, Swing Time was still significantly increased to Pre-OP values at WPO12. *: $p<0.05$ as compared to Pre-OP, ${ }^{* *}: p<0.01$ as compared to Pre-OP. Error bars indicate mean \pm standard error of the mean (SEM). Please click here to view a larger version of this figure. 


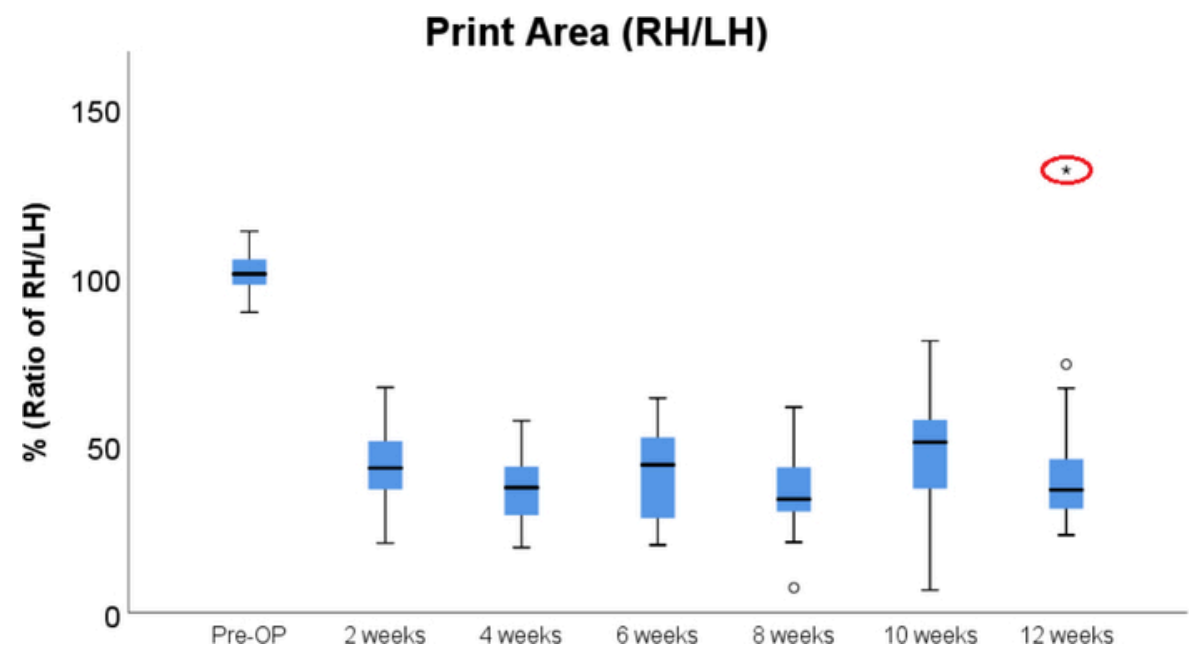

Figure 5: Boxplot of the course of Print Area following sciatic nerve injury. Note the extremum (red ellipse) at WPO12, which is explained by the fact that one animal developed strong contractures of the right hind paw starting from WPO10. The animal was therefore excluded from the statistical analysis displayed in Figure 4. Please click here to view a larger version of this figure.

\section{Femoral nerve neurotmesis}

Femoral nerve resection results in denervation of the quadriceps muscle of the thigh ${ }^{33,34}$. In consequence, knee extension is impaired, resulting in hyperflexion of the ankle joint with consecutive lifting of the paw's heel. Therefore, the respective paw's Print Area (Figure 6B), is strongly reduced after surgery. Print Area of the left hind paw is increased due to a compensatory shifting of weight to the left. This should be kept in mind, as this phenomenon directly influences the calculated ratio between the "experimental" and "control" paw. Starting from WPO4 reinnervation of the quadriceps by the regenerating femoral nerve leads to reversal of these changes resulting in increased Paw Print Area of the right hind paw (Figure 7A). As the quadriceps muscle of the thigh also plays a role in the swing phase of the respective paw, Swing Time (Figure 7B) is greatly prolonged in rats with femoral nerve injury. Mirroring the return of Print Area, Swing Time decreases as the regenerating femoral nerve reaches the quadriceps muscle of the thigh. At WPO10, both parameters of gait returned to baseline, signaling full functional recovery. 


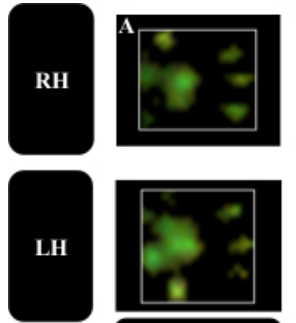

Pre-OP
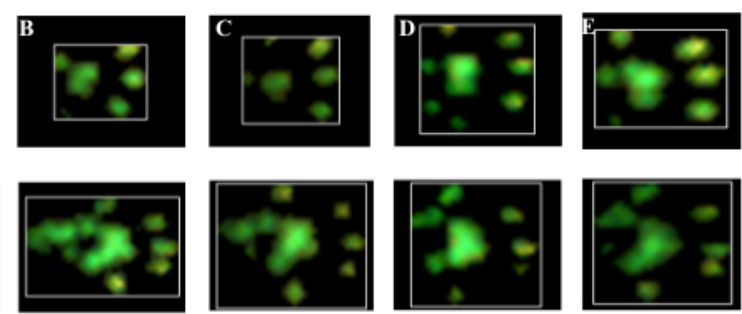

WPO2

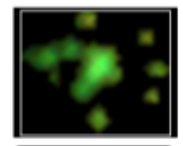

WPO4

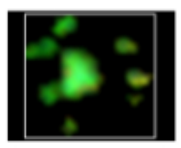

WPO8

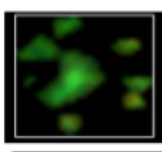

WPO10

Figure 6: Representative paw prints. Representative paw prints prior to $(\mathbf{A})$ and following (B-E) right femoral nerve resection and autograft repair. Print Area of RH decreased strongly at WPO2 (B), while an increase in Print Area of the left hind paw (LH) due to increased weight load became visible. RH Print Area started to increase starting from WPO6 (C) accompanied by a decrease in Print Area of LH. At WPO8 (D) and WPO10 (E) Print Area of RH recovered back close to preoperative levels. (Adapted with permission from Heinzel et al. ${ }^{22}$, licensed under CC BY 4.0.) Please click here to view a larger version of this figure.
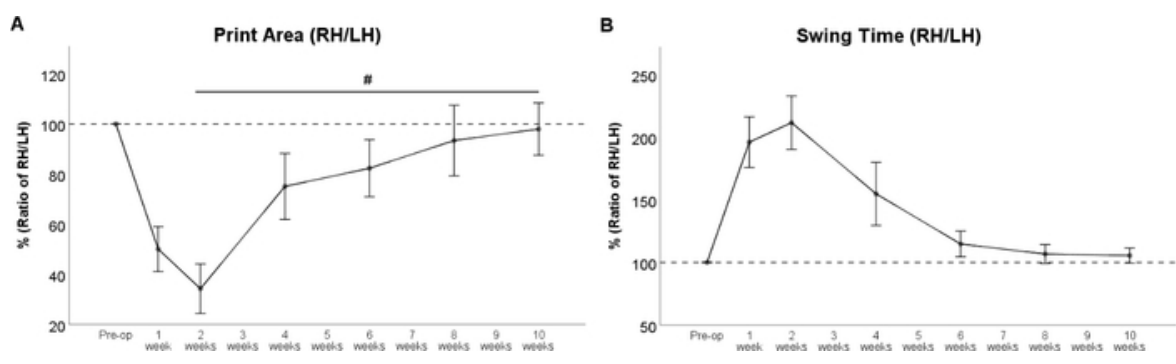

Figure 7: The course of functional recovery after $7-\mathrm{mm}$ resection and autograft repair of the femoral nerve. The course of Print Area Ratio (A) and Swing Time Ratio (B) revealed a strong change immediately after femoral nerve resection, but values recovered back to preoperative values at WPO8. \#: $p<0.05$. Error bars indicate mean \pm SEM. (Adapted with permission from Heinzel et al. ${ }^{22}$, licensed under CC BY 4.0.) Please click here to view a larger version of this figure.

\section{Spinal cord contusion}

Gait analysis revealed markedly altered paw prints after thoracic spinal cord contusion injury (Figure 8), most noteworthy a decrement in Print Area and a marked internal rotation of the hind paws at WPO2 (Figure 8B). Noteworthy, the paw rotation is also implemented as an assessable feature in the BBB, underscoring the applicability of computerized gait analysis to evaluate changes of gait which were originally evaluated with Open Field testing. Regarding the course of the individual gait parameters, spinal cord contusion at the Th11 level resulted in a decrease of the Print Area Ratio (Figure 9A) and increment of Swing Time Ratio (Figure 9B). Both the parameters trended toward baseline levels during the further course of the observation 
period, but there were no statistically significant changes observable. The coordination-related parameter Regularity Index (Figure 9C) also decreased at WPO2, but the degree varied greatly between animals. It also trended toward preoperative values until WPO16. Base of Support of the hind paws (Figure 9D), a general parameter of gait according to Deumens, showed a marked increase, which was statistically significant from WP010 until WPO14. It trended toward baseline levels at WPO16 and was no longer significantly altered from the Pre-OP value at this time point.
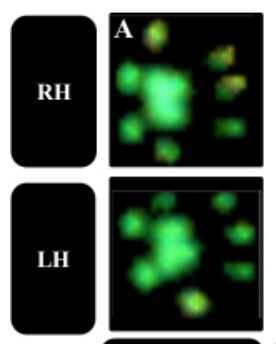

Pre-OP

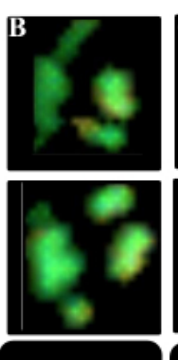

WPO2

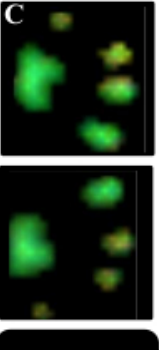

WPO6

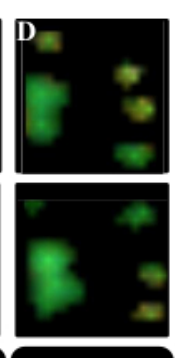

WPO8

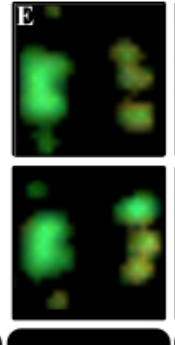

WPO12

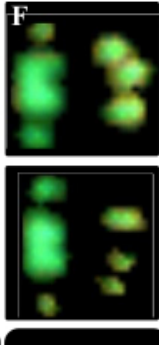

WPO16

Figure 8: Representative paw prints of the two hind paws. Paw prints preoperatively (A) and following thoracic spinal cord contusion injury (B-F). Note the reduction in print area starting from WPO2 (B) accompanied by a notable internal rotation of the paws. During the course of the observation period $(\mathbf{C}-\mathbf{F})$ an increment of the Print Area is observable as well as clearance of the internal rotation. Please click here to view a larger version of this figure. 

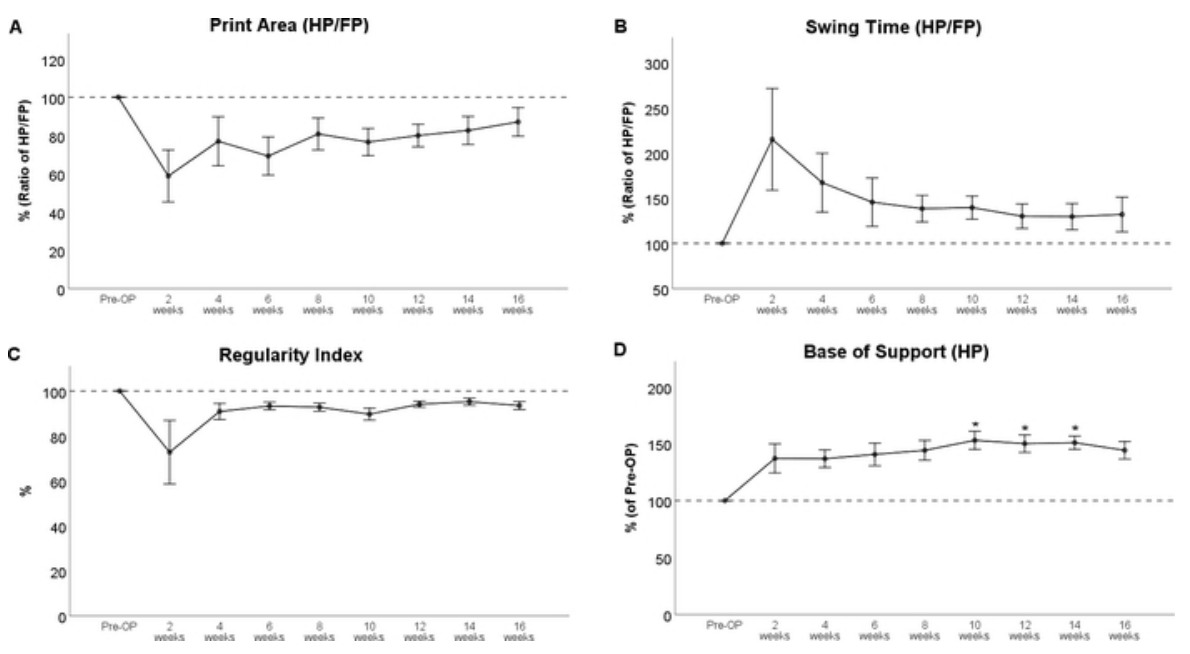

Figure 9: Spinal cord contusion at the Th 11 level. Spinal cord contusion at Th 11 resulted in observable alterations of Print Area Ratio (A) and Swing Time (B) and the Regularity Index (C), but these changes were not statistically significant. Following injury, Base of Support of the hind paws showed a marked increase compared to baseline, what was statistically significant at WPO10 until WPO14. *: $p<0.05$ as compared to Pre-OP. Error bars indicate mean \pm SEM. Please click here to view a larger version of this figure.

Supplementary File 1: Troubleshooting details. Please click here to download this file.

\section{Discussion}

Assessment of functional recovery in animal models of PNI and $\mathrm{SCl}$ remains challenging due to the great variety of evaluation methods, each with individual advantages and disadvantages. Only few approaches have been tested and validated in multiple models of peripheral and central nervous injuries, although promising new techniques which combine motion tracking and machine learning might potentially propel neurobehavioral research to the next level of functional testing. We are convinced that cutting-edge methods broadly applicable to a wide variety of animal and injury models will soon emerge. In the light of these considerations, one of the advantages of AGA is the possibility to evaluate functional recovery in multiple models of nerve injury using only one device. Since the early 2000 s this approach has been used in experimental models of PNI such as the sciatic $^{37}$, peroneal ${ }^{38}$, and femoral nerve injury model ${ }^{22}$ as well as after root avulsion of both the lumbar ${ }^{39}$ and the brachial plexus ${ }^{40}$. Various central nervous injuries including spinal cord contusion injury have also been studied with the method ${ }^{41,42}$. With this paper, we presented a detailed protocol on how to induce three commonly studied nerve injuries as well as how to evaluate functional recovery afterwards. In our opinion, a hands-on-guideline for researchers interested in the area of experimental nerve injury, repair, and regeneration on how to make optimum use of the method's advantageous features would be of great help.

Several authors have addressed the potential of AGA to evaluate functional recovery in rodents, highlighting 
the method's advantage to simultaneously assess gait parameters related to motor and sensory reinnervation 27,28 . Additionally, comparison of data from an experimental paw, e.g., reconstructed nerve injury to an unoperated paw as was shown in both of the models presented allows inclusion of an intra-animal positive control. Inversely, an operated paw without surgical reconstruction or additional treatment could serve as an intra-animal negative control. It was also shown that it is possible to combine AGA with machine learning approaches ${ }^{43}$. In spite of the method's advantages, it also has several limitations and drawbacks, such as the time-consuming training efforts, which are mandatory to accustom the animal to the acquisition procedure 28,44 . Another limitation of AGA is the maximum size of the animals eligible for testing due to the limited dimensions of the apparatus. Therefore, the use of AGA is currently limited to animals the size of rodents and ferrets ${ }^{45}$. Additionally, recently emerging neurobehavioral assessment approaches in the field of motion tracking capable of machine-learning may surpass AGA in both comprehensiveness as well as possible applications ${ }^{18,19,46}$. Most noteworthy, but in accordance with other evaluation methods, it seems that functional recovery as assessed by AGA is strongly limited-if even occurring - in models of sciatic nerve neurotmesis 47,48 . On the other hand, AGA allows for comprehensive evaluation of the course of functional recovery following femoral nerve neurotmesis as shown by our data. With this work, we demonstrated that Paw Print Area is a representative gait parameter assessable via AGA, which is exemplary for the course of functional recovery in the two aforementioned peripheral nerve injury models presented by us. While functional recovery ad integrum was observable after autograft repair of the femoral nerve, AGA parameters were still significantly changed from baseline at the end of the observation period following autograft repair of the sciatic nerve. It is noteworthy in this context that limb contractures are a common phenomenon in rats with sciatic nerve injury and caution is necessary not to confuse these signs of muscular imbalance and paralysis with the proceeding functional recovery ${ }^{32}$. This on the one hand underlines the AGA method's inability to detect significant functional recovery following neurotmesis injury in this model. On the other hand, it raises the question whether it is feasible to evaluate the sciatic nerve injury model of the rat, which is still the most commonly used experimental nerve repair model, by means of gait analysis in general in case the nerve injury is more severe than axonotmesis ${ }^{48}$. Troubleshooting details are provided in Supplementary File 1.

We also provided exemplary data on use of the method to evaluate locomotor function in rats with Spinal Cord Injury, which is possible without any required changes of the hardware setup or acquisition procedure. The same principle applies to other rodent models of central nervous injury $(\mathrm{CNI})^{26,49,50}$ and root avulsion injury. In contrast to isolated PNIs, injuries of the spinal cord are far more complex in their pathophysiological consequences, as a multitude of highly important structures are damaged, involving efferent pathways such as the corticospinal and rubrospinal tracts and afferent pathways such as the dorsal columns and spinothalamic tracts ${ }^{35}$. The challenge to adequately assess these pathological changes is reflected in the comprehensive armamentarium of behavioral tests, such as the Basso, Beattie, and Bresnahan (BBB) score ${ }^{36}$. The gait parameter Base of Support has been reported to increase following central nervous injuries, most probably to account for a resulting instable gait. Base of Support was significantly changed from baseline from WPO10 until WPO14 in our model, supporting our presumption that this parameter allows 
assessment of the course of functional recovery by AGA following thoracic spinal cord contusion injury.

We are convinced that AGA is a feasible tool to evaluate functional recovery in rodents with injuries of the nervous system. Nevertheless, we advise to reflect the observed changes of gait carefully and thoroughly in each respective experimental setup. Alterations in gait parameters, e.g., an increase in Print Area following an immediate postoperative decrement or a decrease in Swing Time proceeding an immediate postoperative elevation of this parameter, over the course of the observation period do not inevitably relate to functional recovery. Instead these changes can also be related to a possible functional adaption to maintain an inconspicuous gait, given that rats are a prey species and try to avoid showing pain or disability to potential predators ${ }^{51}$. It is, therefore, recommended to use automated gait analysis as a complementary tool to relate changes of gait to other outcome measures of peripheral nerve injury and regeneration ${ }^{21}$. As mentioned previously, we also believe that it should be carefully reflected if rodents with sciatic nerve neurotmesis should be investigated by means of AGA as our finding strongly indicates that functional recovery is severely limited in this case.

As shown in our work, AGA's main asset is the possibility to study both motor and sensory reinnervation in a multitude of experimental $\mathrm{PNI}$ models as well as $\mathrm{CNI}$ while requiring only one setup. Therefore, the method is, in our opinion, a highly valuable tool for comprehensive neurobehavioral testing. One of AGA's assets, which is the possibility to study motor and sensory reinnervation in various animal models of $\mathrm{PNI}$ and $\mathrm{CNI}$ while requiring only one setup, is in our opinion the method's main advantage in comparison to other evaluation methods to study functional recovery, such as walking track analysis ${ }^{52}$, Von Frey testing ${ }^{53}$, or gait kinematics ${ }^{16}$. The potential to simultaneously evaluate changes of gait which do either correlate with results of electrophysiological investigations of reinnervated muscle 22 or evaluation methods for sensory function ${ }^{54}$ is promising in regard to future applications of the method. We therefore recommend using AGA to investigate functional recovery in rodent models of forelimb $\mathrm{PNI}$, such as the ulnar, radial, or median nerve, or experimental nerve transfer models ${ }^{55}$, which remain unstudied with this method yet.

We hereby provide a detailed protocol on how to use automated gait analysis to study functional recovery in three rodent models of nerve injury. While the method requires careful consideration of various key aspects such as adequate training and meticulous hard- and software calibration, it is a feasible and valuable complementary tool to evaluate nerve regeneration in rodent models of central and peripheral nerve injury.

\section{Disclosures}

The authors declare that this work was supported by Noldus Inc. by coverage of the open access publication fee. None of the authors received any personal salary or any kind of financial gratuity. The authors have no other competing interests to declare.

\section{Acknowledgments}

The authors would like to thank Karin Brenner for her passionate caretaking of the animals. The authors would also like to thank Claudia Keibl, James Ferguson, Gabriele Leinfellner, and Susanne Drechsler for their assistance during the experimental surgeries.

\section{References}


1. Mohanty, C. B., Bhat, D. I., Devi, B. I. Use of animal models in peripheral nerve surgery and research. Neurology India. 67 (Supplement), S100-S105 (2019).

2. Vela, F. J. et al. Animal models used to study direct peripheral nerve repair: a systematic review. Neural Regeneration Research. 15 (3), 491-502 (2020).

3. Verma, R., Virdi, J. K., Singh, N., Jaggi, A. S. Animals models of spinal cord contusion injury. The Korean Journal of Pain. 32 (1), 12-21 (2019).

4. de Medinaceli, L., Freed, W. J., Wyatt, R. J. An index of the functional condition of rat sciatic nerve based on measurements made from walking tracks. Experimental Neurology. 77 (3), 634-643 (1982).

5. Navarro, X. Functional evaluation of peripheral nerve regeneration and target reinnervation in animal models: a critical overview. The European Journal of Neuroscience. 43 (3), 271-286 (2016).

6. Sperry, R. W. The functional results of muscle transposition in the hind limb of the rat. The Journal of Comparative Neurology. 73 (3), 379-404 (1940).

7. Sperry, R. W. The effect of crossing nerves to antagonistic muscles in the hind limb of the rat. The Journal of Comparative Neurology. 75 (1), 1-19 (1941).

8. Sperry, R. W. Transplantation of motor nerves and muscles in the forelimb of the rat. The Journal of Comparative Neurology. 76 (2), 283-321 (1942).

9. Zimmerman, E. A., Chambers, W. W., Liu, C. N. An experimental study of the anatomical organization of the Cortico-Bulbar System in the Albino Rat. The Journal of Comparative Neurology. 123, 301-323 (1964).

10. Cohen, A. H., Gans, C. Muscle activity in rat locomotion: movement analysis and electromyography of the flexors and extensors of the elbow. Journal of Morphology. 146 (2), 177-196 (1975).

11. Barnett, S. A. The Rat: A Study in Behaviour. Aldine Publishing Company (1963).

12. Hare, G. M. et al. Walking track analysis: utilization of individual footprint parameters. Annals of Plastic Surgery. 30 (2), 147-153 (1993).

13. Hare, G. M. et al. Walking track analysis: a long-term assessment of peripheral nerve recovery. Plastic and Reconstructive Surgery. 89 (2), 251-258 (1992).

14. Bertelli, J. A., Mira, J. C. Behavioral evaluating methods in the objective clinical assessment of motor function after experimental brachial plexus reconstruction in the rat. Journa ofl Neuroscience Methods. 46 (3), 203-208 (1993).

15. Varejao, A. S., Melo-Pinto, P., Meek, M. F., Filipe, V. M., Bulas-Cruz, J. Methods for the experimental functional assessment of rat sciatic nerve regeneration. Neurological Research. 26 (2), 186-194 (2004).

16. Varejao, A. S. et al. Functional assessment of peripheral nerve recovery in the rat: gait kinematics. Microsurgery. 21 (8), 383-388 (2001).

17. Varejao, A. S. et al. Ankle kinematics to evaluate functional recovery in crushed rat sciatic nerve. Muscle and Nerve. 27 (6), 706-714 (2003).

18. Nath, T. et al. Using DeepLabCut for 3D markerless pose estimation across species and behaviors. Nature Protocols. 14 (7), 2152-2176 (2019).

19. Mathis, A. et al. DeepLabCut: markerless pose estimation of user-defined body parts with deep learning. Nature Neuroscience. 21 (9), 1281-1289 (2018). 
20. Deumens, R., Jaken, R. J., Marcus, M. A., Joosten, E. A. The CatWalk gait analysis in assessment of both dynamic and static gait changes after adult rat sciatic nerve resection. Journal of Neuroscience Methods. 164 (1), 120-130 (2007).

21. Heinzel, J. et al. Use of the CatWalk gait analysis system to assess functional recovery in rodent models of peripheral nerve injury - a systematic review. Journal of Neuroscience Methods. 345, 108889 (2020).

22. Heinzel, J. C., Hercher, D., Redl, H. The course of recovery of locomotor function over a 10-week observation period in a rat model of femoral nerve resection and autograft repair. Brain and Behavior. e01580 (2020)

23. Chen, S. $H$. et al. Changes in GABA and $G A B A(B)$ receptor expressions are involved in neuropathy in the rat cuneate nucleus following median nerve transection. Synapse. 66 (6), 561-572 (2012).

24. Chen, S. H. et al. Decreases of glycine receptor expression induced by median nerve injury in the rat cuneate nucleus contribute to NPY release and c-Fos expression. Life Sciences. 90 (7-8), 278-288, (2012).

25. Hetze, S., Romer, C., Teufelhart, C., Meisel, A., Engel, O. Gait analysis as a method for assessing neurological outcome in a mouse model of stroke. Journal of Neuroscience Methods. 206 (1), 7-14 (2012).

26. Sandner, B. et al. Systemic epothilone D improves hindlimb function after spinal cord contusion injury in rats. Experimental Neurology. 306, 250-259 (2018).

27. Kappos, E. A. et al. Validity and reliability of the CatWalk system as a static and dynamic gait analysis tool for the assessment of functional nerve recovery in small animal models. Brain and Behavior. 7 (7), e00723 (2017).

28. Deumens, R., Marinangeli, C., Bozkurt, A., Brook, G. A. Assessing motor outcome and functional recovery following nerve injury. Methods in Molecular Biology. 1162, 179-188 (2014).

29. Webster, G. V. Halstedian principles in the practice of plastic and reconstructive surgery. Stanford Medical Bulletin. 13 (3), 315-316 (1955).

30. Bozkurt, A. et al. A flexible, sterile, and cost-effective retractor system for microsurgery. Microsurgery. 31 (8), 668-670 (2011).

31. Carr, M. M., Best, T. J., Mackinnon, S. E., Evans, P. J. Strain differences in autotomy in rats undergoing sciatic nerve transection or repair. Annals of Plastic Surgery. 28 (6), 538-544 (1992).

32. Dellon, A. L., Mackinnon, S. E. Sciatic nerve regeneration in the rat. Validity of walking track assessment in the presence of chronic contractures. Microsurgery. 10 (3), 220-225 (1989).

33. Jackson, C. M. Anatomy of the rat. By Eunice Chace Greene. With Foreword by Henry H. Donaldson. Transactions of the American Philosophical Society, Philadelphia, New Series, Volume XXVII, 1935, 370 pp., 339 figures. The Anatomical Record. 65 (1), 127-129 (1936).

34. Pena, M. C., Baron, J. Femoral nerve and rectus femoris muscle of the rat: a study in anatomy, histology, and histoenzymes. Annals of Plastic Surgery. 20 (6), 527-532 (1988). 
35. Kjell, J., Olson, L. Rat models of spinal cord injury: from pathology to potential therapies. Disease Models and Mechanisms. 9 (10), 1125-1137 (2016).

36. Basso, D. M., Beattie, M. S., Bresnahan, J. C. A sensitive and reliable locomotor rating scale for open field testing in rats. Journal of Neurotrauma. 12 (1), 1-21 (1995).

37. Bozkurt, A. et al. CatWalk gait analysis in assessment of functional recovery after sciatic nerve injury. Journal of Neuroscience Methods. 173 (1), 91-98 (2008).

38. Godinho, M. J. et al. Immunohistochemical, ultrastructural and functional analysis of axonal regeneration through peripheral nerve grafts containing Schwann cells expressing BDNF, CNTF or NT3. PLoS One. 8 (8), e69987 (2013).

39. Pajenda, G. et al. Spatiotemporally limited BDNF and GDNF overexpression rescues motoneurons destined to die and induces elongative axon growth. Experimental Neurology. 261, 367-376 (2014).

40. Matsuura, Y. et al. The effect of anti-NGF receptor (p75 Neurotrophin Receptor) antibodies on nociceptive behavior and activation of spinal microglia in the rat brachial plexus avulsion model. Spine (Phila Pa 1976). 38 (6), E332-338 (2013).

41. Hamers, F. P., Lankhorst, A. J., van Laar, T. J., Veldhuis, W. B., Gispen, W. H. Automated quantitative gait analysis during overground locomotion in the rat: its application to spinal cord contusion and transection injuries. Journal of Neurotrauma. 18 (2), 187-201 (2001).

42. Koopmans, G. C. et al. The assessment of locomotor function in spinal cord injured rats: the importance of objective analysis of coordination. Journal of Neurotrauma. 22 (2), 214-225 (2005).
43. Frohlich, H., Claes, K., De Wolf, C., Van Damme, X., Michel, A. A machine learning approach to automated gait analysis for the Noldus Catwalk system. IEEE Transaction on Bio-medical Engineering. 65 (5), 1133-1139 (2018).

44. Batka, R. J. et al. The need for speed in rodent locomotion analyses. Anatomical Record (Hoboken, N. J.). 297 (10), 1839-1864 (2014).

45. Wood, T. et al. A ferret model of encephalopathy of prematurity. Developmental Neuroscience. 40 (5-6), 475-489 (2018).

46. Abbas, W., Masip Rodo, D. Computer methods for automatic locomotion and gesture tracking in mice and small animals for neuroscience applications: a survey. Sensors (Basel, Switzerland). 19 (15) (2019).

47. Irintchev, A. Potentials and limitations of peripheral nerve injury models in rodents with particular reference to the femoral nerve. Annals of Anatomy. 193 (4), 276-285 (2011).

48. Haastert-Talini, K. in Modern Concepts of Peripheral Nerve Repair. eds Kirsten Haastert-Talini, Hans Assmus, Gregor Antoniadis. Springer International Publishing. 127-138 (2017).

49. Lu, L. et al. Growth differentiation factor 11 promotes neurovascular recovery after stroke in mice. Frontiers in Cellular Neuroscience. 12 (2018).

50. Caballero-Garrido, E., Pena-Philippides, J. C., Galochkina, Z., Erhardt, E., Roitbak, T. Characterization of long-term gait deficits in mouse dMCAO, using the CatWalk system. Behavioral Brain Research. 331, 282-296 (2017). 
51. Graham, D. M., Hampshire, V. Methods for measuring pain in laboratory animals. Lab Animal. 45 (3), 99-101 (2016).

52. Monte-Raso, V. V., Barbieri, C. H., Mazzer, N., Yamasita, A. C., Barbieri, G. Is the sciatic functional index always reliable and reproducible? Journal of Neuroscience Methods. 170 (2), 255-261 (2008).

53. Stossel, M. et al. Comparative evaluation of chitosan nerve guides with regular or increased bendability for acute and delayed peripheral nerve repair: a comprehensive comparison with autologous nerve grafts and muscle-in-vein grafts. Anatomical Record (Hoboken). 301 (10), 1697-1713 (2018).

54. Vrinten, D. H., Hamers, F. F. 'CatWalk' automated quantitative gait analysis as a novel method to assess mechanical allodynia in the rat; a comparison with von Frey testing. Pain. 102 (1-2), 203-209 (2003).

55. Aman, M., Sporer, M., Bergmeister, K., Aszmann, O. [Animal models for selective nerve transfers of the upper extremity]. Handchirurgie Mikrochirurgie Plastische Chirurgie. 51 (4), 319-326 (2019). 